\title{
Análise da Revisão Cochrane: Erradicação da Helicobacter pylori na Prevenção do Cancro Gástrico. Cochrane Database Syst Rev. 2015;7:CD005583.
}

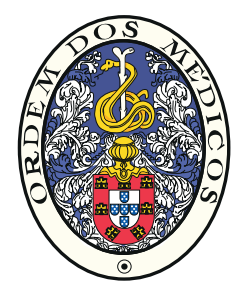

\author{
Analysis of the Cochrane Review: Helicobacter pylori Eradication for the \\ Prevention of Gastric Neoplasia. Cochrane Database Syst Rev. \\ 2015;7:CD005583.
}

\author{
Diogo LIBÂNIO' ${ }^{1}$, Luís Filipe AZEVEDO $\square^{2,3,4}$ \\ Acta Med Port 2015 Nov-Dec;28(6):684-686
}

\section{RESUMO}

A infeção por Helicobacter pylori é um fator de risco para o desenvolvimento de cancro gástrico. A pesquisa e erradicação da Helicobacter pylori na população geral assintomática pode ser considerada para a prevenção primária do cancro gástrico, no entanto a magnitude do benefício e a efetividade desta estratégia ainda não estão completamente esclarecidas. Neste contexto, foi realizada uma revisão sistemática e meta-análise de ensaios clínicos aleatorizados comparando a incidência de cancro gástrico em indivíduos com a infeção que realizaram erradicação e indivíduos infetados que não realizaram a erradicação da Helicobacter pylori. Os resultados dos seis ensaios clínicos incluídos (realizados em países de elevada incidência de cancro gástrico) sugerem que a erradicação da Helicobacter pylori se associa a uma redução relativa do risco de cancro gástrico de $34 \%$. Contudo, a generalização dos resultados para países com menor incidência de cancro gástrico deve ser feita com algum cuidado e a custo-efetividade desta estratégia, neste contexto, permanece ainda incerta.

Palavras-chave: Helicobacter pylori; Infecções por Helicobacter; Neoplasias Gástricas; Revisões Sistemáticas.

\section{ABSTRACT}

Helicobacter pylori infection is a risk factor for gastric adenocarcinoma. Identification of individuals with this infection and its eradication may be considered as a primary prevention strategy to reduce the incidence of gastric adenocarcinoma; however, the magnitude of benefit and the effectiveness of this strategy are still unclear. A systematic review and meta-analysis of randomized clinical trials was conducted comparing the incidence of gastric adenocarcinoma in infected individuals submitted to Helicobacter pylori eradication and individuals not submitted to this therapy. The results of the six included randomized clinical trials (all conducted in countries with high gastric cancer incidence) suggest that Helicobacter pylori eradication is associated with a relative risk reduction of $34 \%$ in gastric cancer incidence. However, generalization of the results to countries with lower gastric cancer incidence should be cautious and the cost-effectiveness of this strategy in this context remains uncertain.

Keywords: Helicobacter Infections; Helicobacter pylori; Stomach Neoplasms; Systematic Reviews.

\section{QUESTÃO CLÍNICA}

Qual o efeito na população em geral da erradicação da Helicobacter pylori em indivíduos infectados na prevenção primária do cancro gástrico? ${ }^{1}$

\section{OBJETIVOS}

Avaliar a efetividade (benefícios e riscos) da erradicação da Helicobacter pylori na população geral assintomática com infeção por $H$. pylori na prevenção do cancro gástrico, nomeadamente quanto à redução da sua incidência $e$ mortalidade.

\section{METODOLOGIA}

Foi realizada uma revisão sistemática e meta-análise de ensaios clínicos aleatorizados (RCTs) nos quais se comparou a incidência de cancro gástrico em doentes assintomáticos com infeção por $H$. pylori submetidos a erradicação da $H$. pylori e em controlos que não realizaram a erradica- ção (placebo ou ausência de tratamento).

A pesquisa bibliográfica (última pesquisa em maio de 2014) foi efetuada em bases de dados electrónicas (Registo Central de ensaios clínicos da Cochrane - CENTRAL, Medline e Embase), foram pesquisadas referências adicionais manualmente, através da pesquisa nas referências dos estudos incluídos e em listas de estudos apresentados em congressos internacionais da área, e finalmente, através da consulta de especialistas.

Foram incluídos RCTs que avaliavam terapêuticas com taxas de erradicação $>50 \%$ e duração da terapêutica de pelo menos uma semana, com um tempo mínimo de seguimento de dois anos e em que a incidência de cancro gástrico fosse reportada (com pelo menos dois casos de cancro reportados). A variável de resultado primária foi a incidência de cancro gástrico, sendo variáveis de resultado secundárias a mortalidade por cancro gástrico, a mortalidade total,

\footnotetext{
1. Serviço de Gastrenterologia. Instituto Português de Oncologia do Porto (IPO-Porto). Porto. Portugal.

2. CINTESIS - Center for Health Technology and Services Research. Faculdade de Medicina. Universidade do Porto. Porto. Portugal.

3. CIDES - Departamento de Ciências da Informação e da Decisão em Saúde. Faculdade de Medicina. Universidade do Porto. Porto. Portugal.

4. Unidade do Porto. Cochrane Portugal. Porto. Portugal.

$\square$ Autor correspondente: Luís Filipe Azevedo. lazevedo@med.up.pt

Recebido: 26 de Outubro de 2015 - Aceite: 26 de Outubro de 2015 | Copyright @ Ordem dos Médicos 2015
} 
a incidência de cancro do esófago e as reações adversas relacionadas com o tratamento. A análise de base realizada teve em conta um método de análise segundo o princípio da intenção-de-tratar modificado (em que foram excluídos indivíduos aleatorizados mas posteriormente confirmados como não elegíveis e indivíduos que não completaram o esquema terapêutico prescrito); no entanto, os autores apresentam também uma análise de sensibilidade complementar, incluindo, entre outros, os resultados de uma análise estritamente segundo o princípio da intenção-de-tratar (considerando todos os indivíduos aleatorizados).

\section{RESULTADOS}

Foram encontrados seis RCTs que cumpriam os critérios de inclusão (cinco estudos publicados e um estudo apresentado em congresso). Em cinco destes estudos o grupo de controlo foi submetido a um placebo e num deles não realizou qualquer tratamento. Cinco dos estudos foram realizados em países asiáticos e um na América do Sul (Colômbia). Em dois destes estudos os indivíduos realizaram concomitantemente suplementos de antioxidantes e vitaminas (tanto no grupo de erradicação como no grupo de controlo). O risco de vieses foi avaliado como baixo, incerto e elevado em três, um e dois estudos respetivamente. $\mathrm{O}$ tempo de follow-up mínimo foi de cinco anos e o máximo de 14,7 anos.

Globalmente, 51 dos 3294 (1,6\%) indivíduos submetidos à erradicação da $H$. pylori e 76 dos 3203 (2,4\%) não submetidos à erradicação foram diagnosticados com cancro gástrico, com uma redução relativa de risco de $34 \%$ associada à erradicação (risco relativo 0,66 - IC 95\% 0,46 - 0,95; Fig. 1). Não foi encontrada heterogeneidade estatisticamente significativa entre os estudos $\left(I^{2}=0\right)$. De acor- do com os resultados combinados, seria preciso tratar 124 indivíduos infetados (IC 95\% 78 - 843) para prevenir um caso de cancro gástrico. É de notar que foram também apresentados os resultados de uma análise de sensibilidade em que foram considerados todos os participantes aleatorizados em cada um dos estudos, uma análise seguindo estritamente o princípio da intenção-de-tratar, tendo sido encontrado um resultado perfeitamente em linha com a análise de base (risco relativo de 0,67 - IC 95\% 0,47 0,95).

Não foram encontradas diferenças significativas na mortalidade por cancro gástrico e mortalidade global entre os grupos, apesar de mortalidade por cancro gástrico menor no grupo da erradicação (Fig. 1). Além disso, não foram encontradas diferenças estatisticamente significativas na incidência de cancro do esófago. Apenas um estudo reportou a incidência de efeitos adversos (maior incidência de rash cutâneo no grupo da erradicação - 3,1\% vs 0,1\%, sem outras diferenças significativas).

$\mathrm{Na}$ análise de subgrupos em que se avaliou separadamente o benefício em doentes com e sem lesões pré-neoplásicas (que os autores definiram como atrofia, metaplasia intestinal ou displasia) não foi encontrado um efeito benéfico significativo da erradicação da $H$. pylori na prevenção do cancro gástrico, apesar da tendência de diminuição da incidência no grupo da erradicação. Adicionalmente, a análise de subgrupos mostrou que o benefício na diminuição da incidência de cancro gástrico era estatisticamente significativo quando considerados os estudos em que os doentes receberam também suplementos de vitaminas e antioxidantes mas não nos estudos em que a intervenção foi unicamente a erradicação da H. pylori.

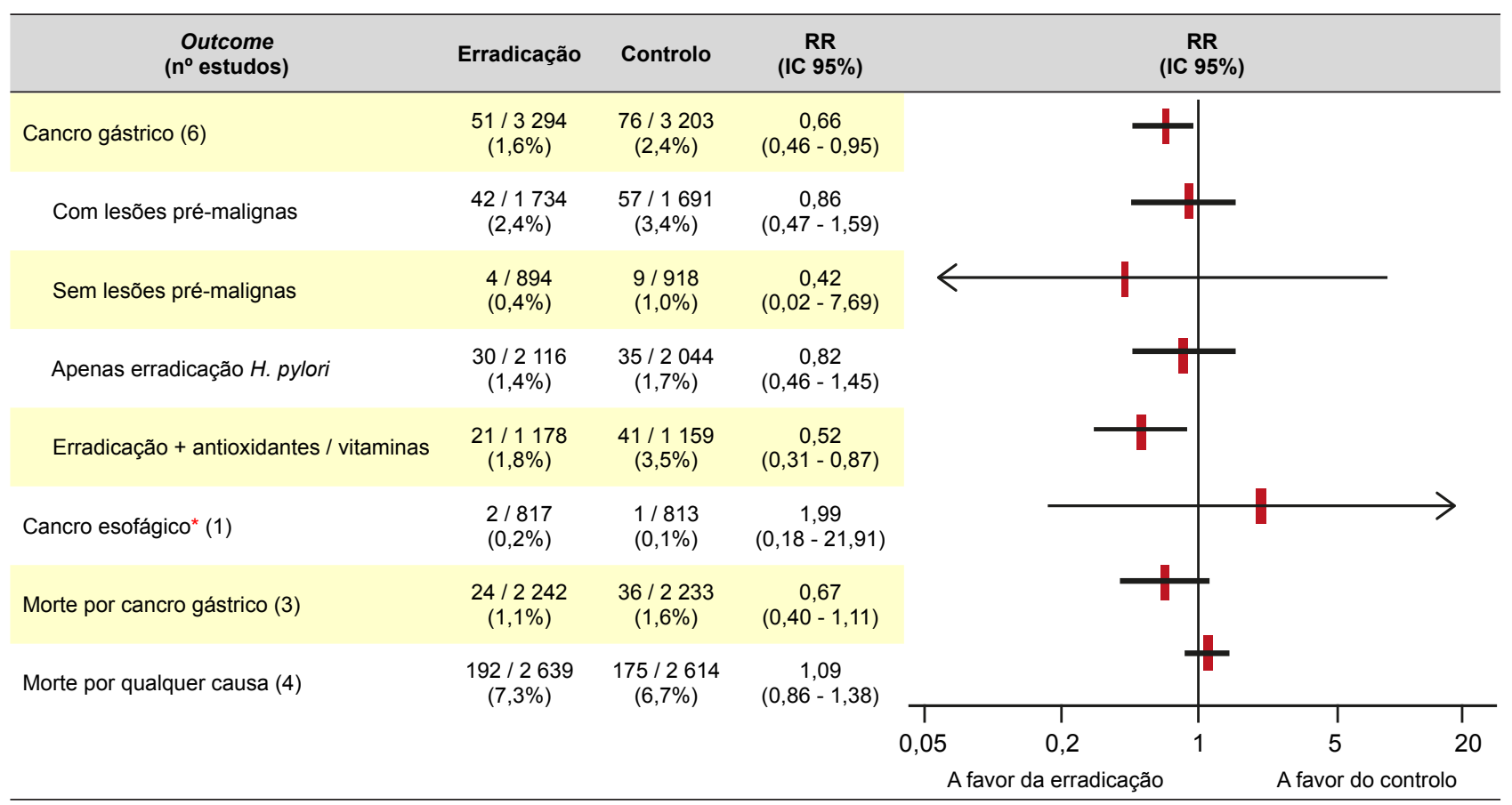

Figura 1 - Sumário dos resultados da meta-análise. Adaptado de Ford et al. ${ }^{1}$ RR: Risco relativo; IC: Intervalo de confiança; ${ }^{*}$ Carcinomas espinocelulares. 


\section{CONCLUSÕES}

A limitada evidência (seis RCTs) existente, de moderada qualidade e proveniente de países com alta incidência de cancro gástrico, sugere que a erradicação da $H$. pylori na população geral assintomática infetada reduz a incidência de cancro gástrico.

\section{COMENTÁRIO}

A $H$. pylori, considerada um carcinogénio tipo I pela OMS ${ }^{2}$ está associada ao desenvolvimento sequencial de gastrite crónica, atrofia, metaplasia intestinal, displasia e adenocarcinoma gástrico do tipo intestinal. ${ }^{3} \mathrm{~A}$ erradicação da $H$. pylori, prevenindo a progressão da carcinogénese gástrica, pode ser uma vista como uma estratégia de prevenção primária na população geral assintomática com esta infeção. Contudo, a elevada prevalência da infeção, os custos e os efeitos adversos da terapêutica de erradicação suscitam dúvidas quanto à custo-efetividade desta estratégia.

A revisão sistemática e meta-análise apresentada sugere que a erradicação da $H$. pylori na população geral assintomática infetada diminui a incidência de cancro gástrico. Os resultados devem ser no entanto interpretados com precaução. Por um lado, cinco dos seis estudos incluídos provêm de países asiáticos com alta incidência de cancro gástrico, pelo que fica por esclarecer o benefício em países com incidência baixa ou moderada (caso de Portugal). Além disso, a análise de subgrupos apenas mostrou benefício estatisticamente significativo nos estudos em que foram realizadas co-intervenções como a suplementação com antioxidantes ou vitaminas, sugerindo que estas co-intervenções podem ter um efeito benéfico aditivo à erradicação. Além disso, foram incluídos doentes com cancro gástrico de tipo difuso, nos quais o papel da $H$. pylori não se encontra bem estabelecido.

Por outro lado, algumas características dos estudos incluídos e aspetos metodológicos da revisão sistemática podem ter subestimado o real benefício da erradicação. Foram incluídos estudos que utilizaram esquemas de erradicação com eficácia $>50 \%(55,6 \%-73,2 \%$ nos estudos incluídos, considerada sub-ótima) e não foi realizada análise separada do grupo em que a erradicação foi eficaz (ou seja, a inclusão de doentes submetidos a erradicação nos quais esta não foi eficaz pode ter subestimado o benefício). Por outro lado, o endpoint primário definido foi a incidência de cancro gástrico. Contudo, a displasia, que é considerada uma lesão pré-maligna que deve ser submetida a resseção (idealmente endoscópica, cirúrgica em alternativa) sempre que haja uma lesão visível, é também um endpoint clinicamente relevante pelo que a incidência de lesões displásicas seria também um endpoint clinicamente relevante. Por último, dada a evolução temporal longa desde a infeção até ao outcome final a evitar (cancro gástrico), é expectável que a erradicação da $H$. pylori tenha os seus maiores benefícios décadas após a erradicação, pelo que tempos de seguimento maiores poderão esclarecer definitivamente o real benefício.

\section{IMPLICAÇÕES PARA A PRÁTICA CLÍNICA}

Os resultados deste estudo sugerem que a erradicação da Helicobacter pylori diminui a incidência de cancro gástrico, pelo menos em países com elevada incidência de cancro gástrico. No caso de Portugal (moderada incidência), a erradicação da $H$. pylori pode ter um papel na prevenção primária em indivíduos assintomáticos apesar da custo-efetividade desta estratégia ser incerta.

\section{REFERÊNCIAS}

1. Ford AC, Forman D, Hunt R, Yuan Y, Moayyedi P. Helicobacter pylori eradication for the prevention of gastric neoplasia. Cochrane Database Syst Rev; 2015: CD005583.

2. Herrera V, Parsonnet J. Helicobacter pylori and gastric adenocarcinoma.

Clin Microbiol Infect. 2009;15:971-6

3. Correa P. A human model of gastric carcinogenesis. Cancer Res. 1988.48:3554-60.

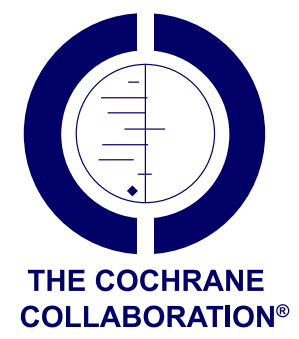




\section{Análise da Revisão Cochrane: Erradicação da Helicobacter pylori na Prevenção do Cancro Gástrico. Cochrane Database Syst Rev. 2015;7:CD005583.}

Acta Med Port 2015:28:684-686

Publicado pela Acta Médica Portuguesa, a Revista Científica da Ordem dos Médicos

Av. Almirante Gago Coutinho, 151

1749-084 Lisboa, Portugal.

Tel: +351218428215

E-mail: submissao@actamedicaportuguesa.com

www.actamedicaportuguesa.com

ISSN:0870-399X | e-ISSN: 1646-0758

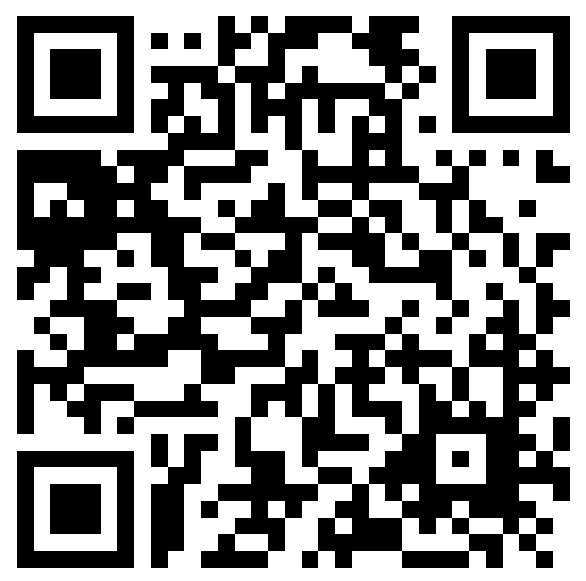

